\title{
PBEF promotes the apoptosis of pulmonary microvascular endothelial cells and regulates the expression of inflammatory factors and AQP1 through the MAPK pathways
}

\author{
GUANG-FENG MING ${ }^{1}$, XIN-HUA MA ${ }^{1}$, DAO-MIAO XU ${ }^{1}$, ZHI-YONG LIU ${ }^{1}$, \\ YU-HANG AI ${ }^{1}$, HUI-XIA LIU ${ }^{2}$ and ZAN-HUA SHI ${ }^{3}$ \\ Departments of ${ }^{1}$ Critical Care Medicine, ${ }^{2}$ Geriatrics, and ${ }^{3}$ Neurosurgery, \\ Xiangya Hospital, Central South University, Changsha, Hunan 410008, P.R. China
}

Received January 22, 2015; Accepted July 7, 2015

DOI: $10.3892 / \mathrm{ijmm} .2015 .2283$

\begin{abstract}
Pre-B cell colony-enhancing factor (PBEF) has been shown to have a variety of biological functions. Studies have proven that PBEF plays a functional role in acute lung injury (ALI). Therefore, in this study, we aimed to confirm the importance of PBEF in ALI. The effects of PBEF overexpression on the apoptosis of human pulmonary microvascular endothelial cells (HPMECs) were analyzed by flow cytometry, and the results indicated that PBEF promoted the apoptosis of HPMECs, which aggravated the development of ALI. Comparative experiments involving increasing and decreasing PBEF expression demonstrated that PBEF promoted the expression of inflammatory factors, such as interleukin (IL)-1 $\beta$, IL- 6 and IL- 8 in the HPMECs , thus intensifying the inflammatory response. PBEF also inhibited the expression of aquaporin 1 (AQP1), which caused a dysfunction and imbalance in water transport. Moreover, we also found that tumor necrosis factor (TNF)- $\alpha$ promoted the expression of PBEF in the HPMECs. After blocking the mitogen-activated protein kinase (MAPK) and phosphoinositide 3-kinase (PI3K) pathways, we found that PBEF regulated the expression of inflammatory factors and AQP1, mainly through the MAPK pathways. Taken together, these results demonstrate that the increase in intracellular PBEF expression promoted the apoptosis of HPMECs and the expression of inflammatory factors and thus enhanced the inflammatory response and inhibited the expression of AQP1, which resulted in abnormal water transport, diminishing the regulatory effects of AQP1 on water transport.
\end{abstract}

Correspondence to: Dr Zan-Hua Shi, Department of Neurosurgery, Xiangya Hospital, Central South University, 87 Xiangya Road, Kaifu, Changsha, Hunan 410008, P.R. China

E-mail: bright900@163.com

Key words: acute lung injury, pre-B cell colony-enhancing factor, aquaporin 1, p38, human pulmonary microvascular endothelial cells

\section{Introduction}

Acute lung injury (ALI), a clinical syndrome caused by a variety of non-cardiogenic factors, is characterized by increased capillary permeability, pulmonary edema, atelectasis and refractory hypoxemia and is induced by injury to pulmonary capillary endothelial cells and alveolar epithelial cells; ALI has the potential to develop into acute respiratory distress syndrome (ARDS), which is associated with a mortality rate of 40-60\% worldwide (1). ALI that manifests clinically as ARDS is a major cause of multiple organ dysfunction syndrome (MODS) (1). Inflammatory mediators, such as interleukin (IL)-1 $\beta$ and IL-8, play key roles in the pathogenesis of ARDS, which is the primary cause of mortality in patients with these conditions.

Pre-B cell colony-enhancing factor (PBEF) is a highly conserved 52-kDa protein (2). It was originally cloned from a complementary DNA (cDNA) library of activated human peripheral blood mononuclear cells (PBMCs) and was identified as a secreted protein that enhances the effects of stem cell factor and IL-7 on pre-B cell colony formation. It is now evident that $\mathrm{PBEF}$ is a multifunctional protein, having nicotinamide phosphoribosyltransferase, adipokine and cytokine activities. Experimental and clinical analyses on the inflammatory aspects of PBEF conducted by Moschen et al (2) demonstrated that PBEF exhibited inflammatory and immune stimulatory activity, and that it regulated the expression of several inflammatory factors, such as tumor necrosis factor (TNF)- $\alpha$, IL-1 $\beta$ and IL-6. Ye et al (3) found that the level of PBEF in bronchoalveolar lavage fluid and blood plasma of animal and patients with ALI was significantly increased, suggesting that PBEF may be an indicator of lung injury. Bajwa et al (4) confirmed that the PBEF gene polymorphism was closely related to the prognosis of ARDS. Taken together, these data indicate that PBEF plays an important role in ALI. Ye et al (5) demonstrated that PBEF played an important role in the loss of barrier function in pulmonary vascular endothelial cells. Liu et al (6) found that IL-1 $\beta$ promoted the expression of PBEF in pulmonary vascular endothelial cells, and that the secretion of inflammatory factors [IL-8, IL-16 and chemokine (C-C motif) receptor 3 (CCR3)] was involved. 
However, the exact mechanisms of PBEF-related pulmonary vascular endothelial cell injury remain unclear. Studies have proven that mitogen-activated protein kinases (MAPKs) are involved in the pathological process of lung injury $(2,7,8)$; however, the association between the signal transduction pathways of the PBEF pro-inflammatory response in ALI and MAPK pathways remains unclear.

Researchers have indicated that aquaporins (AQPs) play an important role in the pathogenesis of ALI $(9,10)$. Studies have focused on vascular endothelial permeability; however, little information on the effects of the fluid-transport function of AQPs in the formation of pneumonedema is available. Furthermore, the expression of AQPs in lung tissue in ALI remains a controversial topic. Certain studies have demonstrated a decrease in AQP expression in patients suffering from ALI (11,12), whereas in another study, Lai et al (13) demonstrated that inflammatory factors promoted the expression of AQP1 in vitro. In the present study, PBEF was upregulated using an overexpression plasmid, and TNF- $\alpha$ and siRNA were also used to investigate the association of PBEF with inflammatory factors and AQP activity. The mechanisms of ALI were further examined with the use of MAPK inhibitors. Our study of the pathological mechanisms of ALI provides information which may prove to be useful in the prevention and treatment of ALI.

\section{Materials and methods}

Cell line and cell culture. Human pulmonary microvascular endothelial cells (HPMECs) were purchased from the American Type Culture Collection (ATCC, Manassas, VA, USA) and cultured in DMEM medium supplemented with $10 \%$ fetal bovine serum (FBS; both from Gibco, Paisley, UK), $100 \mathrm{U} / \mathrm{ml}$ penicillin and $50 \mathrm{U} / \mathrm{ml}$ streptomycin (both from Sigma-Aldrich China, Inc., Shanghai, China) at $37^{\circ} \mathrm{C}$ in an incubator with $5 \% \mathrm{CO}_{2}$.

Construction of the PBEF-overexpressing plasmid. The PBEFoverexpressing plasmid was constructed by inserting the cDNA clone of PBEF reverse transcriptase from the HPMECs into the pEGFP-N1 vector [from Takara Biotechnology (Dalian) Co., Ltd., Dalian, China]. Total RNA was extracted using TRIzol reagent (Shanghai Invitrogen Biotechnology Co., Ltd., Shanghai, China) to prepare the cDNA clone of PBEF. Reverse transcription polymerase chain reaction (RT-PCR) was performed to retrieve the full-length mouse PBEF cDNA using cDNA and a reverse transcription kit (Promega Biotech Co., Ltd., Beijing, China). The PCR conditions were as follows: a pre-denaturation step at $94^{\circ} \mathrm{C}$ for $5 \mathrm{~min}$, followed by $94^{\circ} \mathrm{C}$ for $30 \mathrm{sec}, 58^{\circ} \mathrm{C}$ for $30 \mathrm{sec}$, $72^{\circ} \mathrm{C}$ for $90 \mathrm{sec}$ for 30 cycles, and finally a complete cycle at $72^{\circ} \mathrm{C}$ for $10 \mathrm{~min}$. The amplified products were analyzed by electrophoresis, and the specific 1,473-bp band of the amplified cDNA fragment was confirmed and collected. The cloned PBEF cDNA was inserted into the pEGFP-N1 vector with XhoI and BamHI [Takara Biotechnology (Dalian) Co., Ltd.], sequenced and verified. The primers used for PBEF amplification were: PBEF-F, 5'-CCGCTCGAGATGAATCCTGCGGCAGA AGC-3' and PBEF-R, 5'-CGGGATCCCGATGATGTGCT GCTTCCAGTTC-3'. Underlined primers indicate the restriction enzyme site.
Table I. siRNA sequences

\begin{tabular}{lc}
\hline Name & \multicolumn{1}{c}{ Sense/antisense siRNA $\left(5^{\prime} \rightarrow 3^{\prime}\right)$} \\
\hline PBEF-homo-514 & GGCCAAAUAUUUGUUAGAATT \\
& UUCUAACAAAUAUUUGGCCTT \\
PBEF-homo-1056 & GGGAUGGAGUAGAUAUUAATT \\
& UUAAUAUCUACUCCAUCCCTT \\
PBEF-homo-1279 & GGGCCGAUUAUCUUUACAUTT \\
& AUGUAAAGAUAAUCGGCCCTT \\
Negative control & UUCUCCGAACGUGUCACGUTT \\
& ACGUGACACGUUCGGAGAATT
\end{tabular}

PBEF, pre-B cell colony-enhancing factor.

Design and synthesis of siRNA. The PBEF-targeting siRNA was designed and synthesized by Shanghai GenePharma Co., Ltd. (Shanghai, China). The siRNA sequences are presented in Table I.

Cell transfection and induction of PBEF expression by TNF- $\alpha$. Cell transfection was carried out using Lipofectamine 2000 according to the manufacturer's instructions (Shanghai Invitrogen Biotechnology Co., Ltd.). Briefly, the cells were seeded in 6-well plates at a concentration of $1 \times 10^{5}$ cells $/ \mathrm{ml}$ $(1 \mathrm{ml} /$ well). When the cells reached $70 \%$ confluency $24 \mathrm{~h}$ after seeding, the plasmid (pEGFP-N1-PBEF) and PBEF siRNA were transfected into the cells at final concentrations of $4 \mu \mathrm{g} / \mathrm{ml}$ and $50 \mathrm{nM}$, respectively. The medium was changed 4-6 h after transfection. TNF- $\alpha$ (Peprotech, Inc., Rocky Hill, NJ, USA) was added at a concentration of $5.75 \mathrm{nM}$, as previously described (14), and was re-added every $24 \mathrm{~h}$. The effects of the induction of PBEF expression were detected by reverse transcription-quantitative PCR (RT-qPCR) and western blot anlaysis after $72 \mathrm{~h}$.

Determination of cell apoptosis. Cell apoptosis was evaluated by co-staining of the cells with Annexin V-FITC and propidium iodide (PI; BD Pharmingen, San Diego, CA, USA). Briefly, the cells were collected $72 \mathrm{~h}$ after transfection and resuspended in $0.5 \mathrm{ml}$ binding buffer and stained with $5 \mu \mathrm{l}$ Annexin V-FITC and $5 \mu \mathrm{l}$ PI, and the solution was then incubated for $15 \mathrm{~min}$ at room temperature in the dark. Thereafter, the cells were immediately analyzed on a Coulter Epics XL Flow Cytometer (Beckman Coulter, Inc., Brea, CA, USA). The fluorochrome was excited at $488 \mathrm{~nm}$ by an argon ion laser, and Annexin V and PI emissions were monitored at 525 and $630 \mathrm{~nm}$, respectively. In each analysis, 10,000 events were recorded. The dual parametric dot plots were used to calculate the percentage of non-apoptotic viable cells in the lower left quadrant (Annexin V-negative/PI-negative), early apoptotic cells in the lower right quadrant (Annexin V-positive/PI-negative), late apoptotic cells in the upper right quadrant (Annexin V-positive/PI-positive) and necrotic cells in the upper left quadrant (Annexin V-negative/PI-positive).

Blocking MAPK and AKT signaling pathways. SB 203580 (a p38 inhibitor), PD 98059 [an extracellular signal- 
Table II. Primers used for quantitative PCR.

\begin{tabular}{llcc}
\hline Primer name & \multicolumn{1}{c}{ Primer sequences $\left(5^{\prime} \rightarrow 3^{\prime}\right)$} & Product size (bp) & \multicolumn{1}{c}{$\begin{array}{c}\text { GenBank } \\
\text { accession no. }\end{array}$} \\
\hline $\begin{array}{l}\text { PBEF-F } \\
\text { PBEF-R }\end{array}$ & $\begin{array}{l}\text { TGCTACAGAAGTTGACAAGAGATC } \\
\text { CCCTGCTGGCGTCCTATG }\end{array}$ & 102 & U02020 \\
IL-1 $\beta-F$ & TGGCTTATTACAGTGGCAATG & 134 & NM_000576 \\
IL-1 $\beta-R$ & GTGGTGGTCGGAGATTCG & 104 & NM_000600 \\
IL-6-F & TAGGACTGGAGATGTCTGAG & & \\
IL-6-R & GTGGAGAAGGAGTTCATAGC & 85 & NM_000584 \\
IL-8-F & CACAAACTTTCAGAGACAGCAGAG & 100 & NM_001185060 \\
IL-8-R & ACACAGTGAGATGGTTCCTTCC & & \\
AQP1-F & ACTCATCTACGACTTCATCC & 97 & NM_002046 \\
AQP1-R & GGCATCCAGGTCATACTC & & \\
GAPDH-F & TCTCTGCTCCTCCTGTTC & & \\
GAPDH-F & ACTCCGACCTTCACCTTC &
\end{tabular}

PBEF, pre-B cell colony-enhancing factor; IL, interleukin; AQP1, aquaporin 1.

regulated kinase (ERK)1/2 inhibitor], c-Jun N-terminal kinase (JNK) inhibitor II and LY 294002 [a phosphoinositide 3-kinase (PI3K) inhibitor] were all purchased from Calbiochem (Merck Millipore, Shanghai, China). The cells were exposed to the inhibitors at a final concentration of $20 \mu \mathrm{M}$ for $1 \mathrm{~h}$ prior to transfection and induction of epression. DMSO (Sigma-Aldrich China, Inc.) was used as a negative control, as previously described (15).

$R T-q P C R$. Total RNA was extracted using TRIzol reagent (Shanghai Invitrogen Biotechnology Co., Ltd.) after the cells had been collected and quantitified. This was followed by reverse transcription with $1 \mu \mathrm{g}$ RNA. Quantitative (real-time) PCR was performed using the SYBR-Green dye method (SYBR-Green PCR Master Mix; Toyobo Co., Ltd., Osaka, Japan) under the follows conditions: $95^{\circ} \mathrm{C}$ for $5 \mathrm{~min}, 95^{\circ} \mathrm{C}$ for $30 \mathrm{sec}, 55^{\circ} \mathrm{C}$ for $30 \mathrm{sec}, 72^{\circ} \mathrm{C}$ for $30 \mathrm{sec}$, for 40 cycles. The reaction mixture $(35 \mu \mathrm{l})$ contained $100 \mathrm{ng}$ cDNA $(20 \mu \mathrm{l})$. The primers used are presented in Table II. The experiments were repeated 3 times and analyzed by comparing the $2^{-\Delta \Delta C t}$ values.

Western blot anlaysis. Total cellular proteins were extracted by incubating the cells in lysis buffer obtained from Cell Signaling Technology, Inc. (Beverly, MA, USA). The protein concentrations of the cell lysates were determined using a bicinchoninic acid assay kit (Pierce Biotechnology, Inc., Rockford, IL, USA) according to the manufacturer's instructions. For SDS-PAGE, $10 \%$ gels were used, and equal amount of proteins were loaded per lane. Following electrophoresis, the separated proteins were transferred onto nitrocellulose membranes (Pierce Biotechnology, Inc.) and blocked with 5\% non-fat milk in TBST buffer for $1 \mathrm{~h}$. Subsequently, the membranes were incubated with primary antibodies [anti-PBEF (ab45890), anti-IL-1 $\beta$ (ab9722), anti-IL-6 (ab6672), anti-IL-8 (ab7747), anti-AQP1 (ab15080) and anti-GAPDH (ab181602) antibodies; Abcam, Shanghai, China] at a 1:1,000 dilution in 5\% non-fat milk overnight at $4^{\circ} \mathrm{C}$ followed by secondary antibodies [goat anti-rabbit IgG H\&L (HRP); ab97051; Abcam] at a 1:2,000 dilution for $1 \mathrm{~h}$ at room temperature. Protein bands were visualized on X-ray film using an enhanced chemiluminescence system (Pierce Biotechnology, Inc.).

Statistical analysis. Experiments were carried out at least in triplicate, and the results are expressed as the means \pm SD. Statistical analysis was performed using the SPSS statistical package (SPSS 17.0 for Windows; SPSS, Inc., Chicago, IL, USA). The differences between 2 groups were analyzed using a two-tailed Student's t-test, and those between 3 or more groups were analyzed by one-way analysis of variance (ANOVA) multiple comparisons. A P-value $<0.05$ was considered to indicate a statistically significant difference.

\section{Results}

Regulation of the intracellular expression level of PBEF. RT-qPCR was employed to evaluate the inhibitory effects of siRNA, and the results revealed that siRNA PBEF-homo-1279 inhibited PBEF gene expression by up to $85 \%$ at a concentration of $50 \mathrm{nM}$ (data not shown). The results of RT-qPCR and western blot anlaysis revealed that the PBEF-targeting siRNA significantly inhibited the mRNA and protein expression of PBEF (Fig. 1). Moreover, transfection of the cells with the PBEF-overexpressing plasmid (pEGFP-N1-PBEF) increased the expression level of PBEF to a level 10-fold greater than that of the control group (Fig. 1). These results indicated the powerful effects of the PBEF-overexpressing plasmid and PBEF-targeting siRNA on the expression of PBEF. The effects of TNF- $\alpha$ on the overexpression and knockdown of PBEF were also investigated, and the results revealed that the expression of PBEF increased significantly with the presence of TNF- $\alpha$, which indicated that TNF- $\alpha$ promoted the expression of PBEF in the HPMECs (Fig. 2). The effects of TNF- $\alpha$ on the expression of PBEF were further enhanced by combining TNF- $\alpha$ and transfection with the PBEF-overexpressing plasmid. However, 

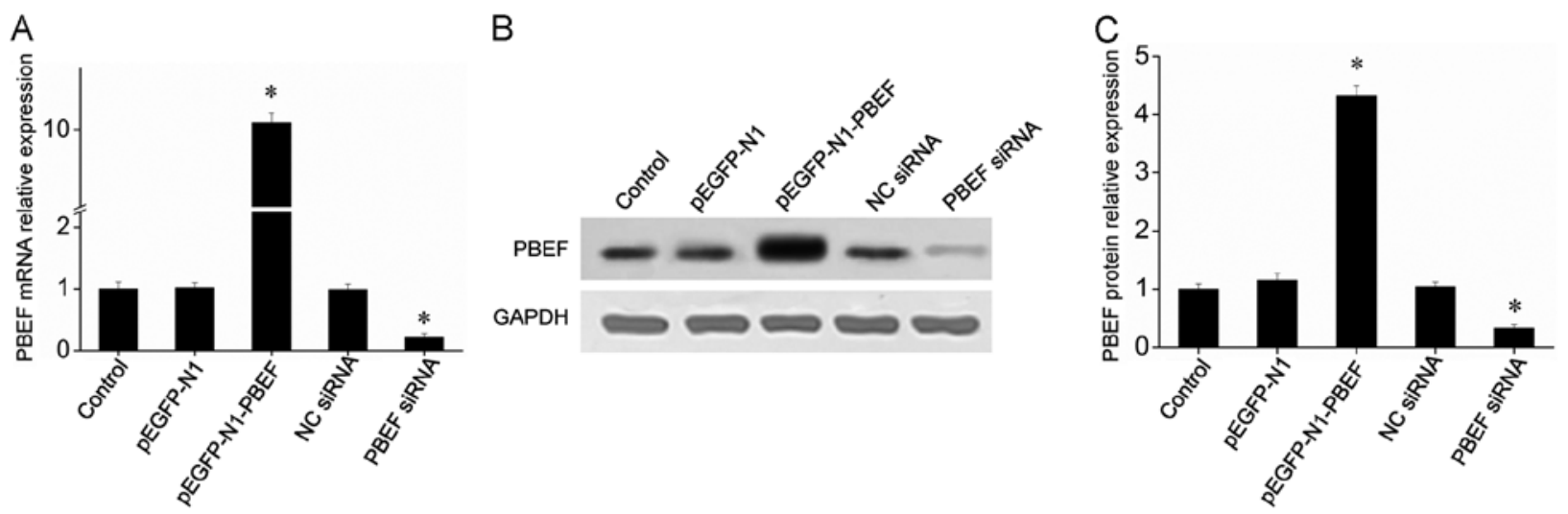

Figure 1. Overexpression and silencing of pre-B cell colony-enhancing factor (PBEF). (A) The changes in PBEF mRNA expression were detected by RT-qPCR (B and C) The changes in PBEF protein expression were detected by western blot analysis. Cells were seeded in 6-well plates at a concentration of 1x10 5 cells/ $\mathrm{ml}(1 \mathrm{ml} /$ well). When the cells reached $70 \%$ confluency $24 \mathrm{~h}$ after seeding, the plasmid (pEGFP-N1-PBEF) and PBEF siRNA were transfected into the cells at final concentrations of $4 \mu \mathrm{g} / \mathrm{ml}$ and $50 \mathrm{nM}$, respectively. The medium was changed $4-6 \mathrm{~h}$ after transfection. GAPDH served as a control for the equal loading of proteins. Each bar represents the means \pm SD from 3 samples ( $(\mathrm{P}<0.05$ vs. control). NC, negative control.

A

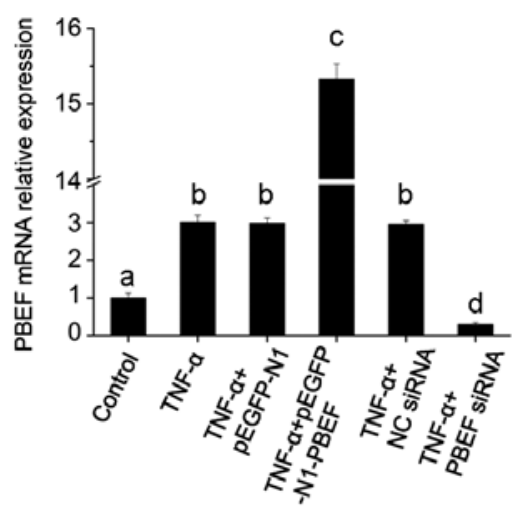

B

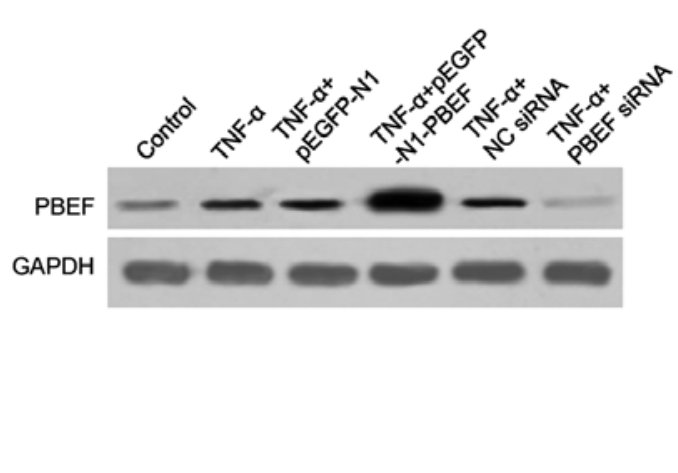

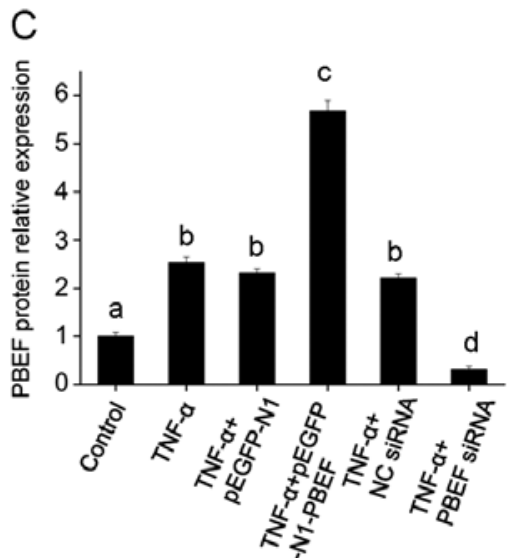

Figure 2. Effects of tumor necrosis factor (TNF)- $\alpha$ on pre-B cell colony-enhancing factor (PBEF) expression. TNF- $\alpha$ was added at a concentration of $5.75 \mathrm{nM}$, and then re-added every $24 \mathrm{~h}$. The effect of the induction of PBEF expression was detected by (A) RT-qPCR and (B and C) western blot analysis after $72 \mathrm{~h}$. GAPDH served as a control for the equal loading of proteins. Each bar represents the means \pm SD from 3 samples, and bars labeled with diffent letters (a-d) indicate a statistically significant difference between groups ("P<0.05 vs. the control). NC, negative control.

the TNF- $\alpha$-induced overexpression of PBEF was blocked by PBEF siRNA (Fig. 2). Taken together, our results suggest that TNF- $\alpha$ has an enhancing effect when combined with the PBEF-overexpressing plasmid and an antagonistic effect when combined with PBEF siRNA.

Overexpression of PBEF promotes HPMEC apoptosis. To examine the effects of PBEF overexpression on HPMEC apoptosis, flow cytometric analysis was employed on the cells co-stained with Annexin V-FITC and PI. The results revealed that the overexpression of PBEF significantly promoted both the early- and late-phase apoptosis of the HPMECs (Fig. 3). These results suggest that an increase in the PBEF expression level causes apoptotic cell death, and this in turn, may lead to the development of ALI.

$P B E F$ regulates the expression of inflammatory factors and $A Q P s$. To examine the effects of PBEF expression on inflammatory factors and AQPs, RT-qPCR and western blot analysis were employed to measure the mRNA and protein levels of PBEF in the HPMECs. The results indicated that PBEF overexpression significantly increased the mRNA and protein expression of IL- $1 \beta$, IL- 6 and IL- 8 , and this was further enhanced by the addition of TNF- $\alpha$ (Fig. 4). The mRNA and protein expression of AQP1 was inhibited by the overexpression of PBEF and this effect was enhanced by TNF- $\alpha$ (Fig. 4). Furthermore, the inhibition of PBEF using siRNA downregulated the mRNA and protein levels of IL-1 $\beta$, IL- 6 and IL-8 and increased the mRNA and protein level of AQP1. Taken together, these results indicate that PBEF enhances the mRNA and protein expression of IL-1 $\beta$, IL- 6 and IL-8, and inhibits the mRNA and protein expression of AQP1.

PBEF regulates the expression of inflammatory factors and $A Q P$ s through the MAPK pathways. The regulatory effects of MAPKs on inflammatory factors and AQPs have previously been demonstrated (16). Therefore, in this study, experiments were also carried out to investigate the association between the PBEF-regulated expression of inflammatory factors and AQPs, and the activation of the MAPK and PI3K pathways. 

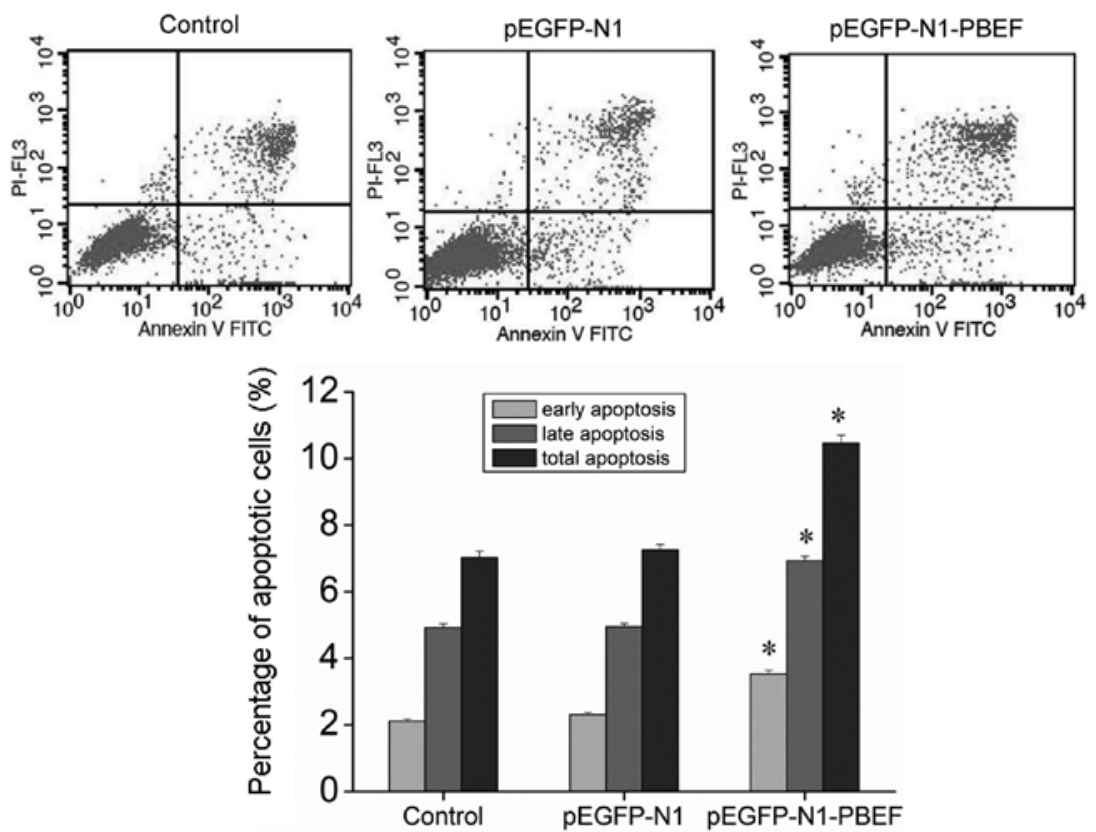

Figure 3. Effect of pre-B cell colony-enhancing factor (PBEF) expression on human pulmonary microvascular endothelial cell (HPMEC) apoptosis, as measured by Annexin V-FITC-PI staining and flow cytometric analysis. The results are presented as the means $\pm \mathrm{SD},(\mathrm{n}=3) ;{ }^{*} \mathrm{P}<0.05$ vs. control.
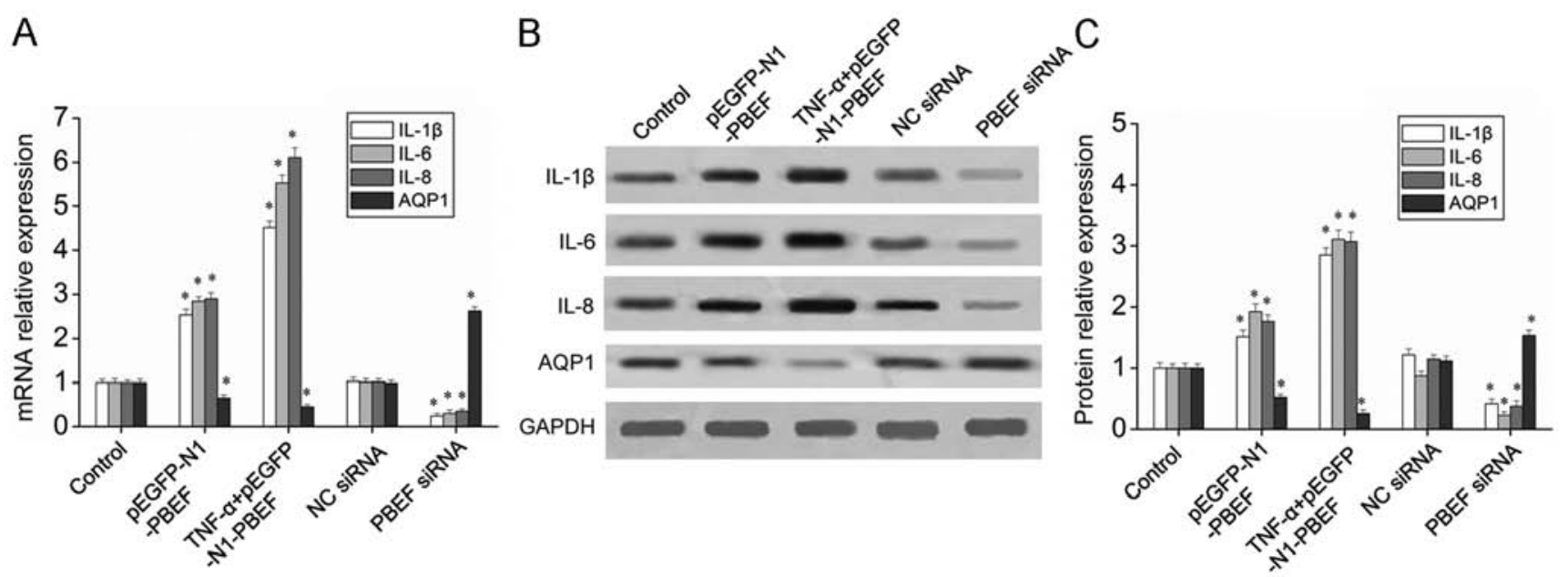

Figure 4. Effect of pre-B cell colony-enhancing factor (PBEF) expression on inflammatory factor and aquaporin (AQP)1 expression. The expression of interleukin (IL)-1 1 , IL-6, IL-8 and AQP1 at the (A) mRNA and (B and C) protein level was detected by RT-qPCR and western blot analysis, respectively. GAPDH served as a control for the equal loading of proteins. Each bar represents the means \pm SD from 3 samples (" $\mathrm{P}<0.05$ vs. control).

Inhibitors of several signaling pathways (p38, ERK, JNK and $\mathrm{PI} 3 \mathrm{~K})$ at non-cytotoxic concentrations $(20 \mu \mathrm{M})$ were introduced into PBEF-overexpressing HPMECs that were treated or not with TNF- $\alpha$. The mRNA and protein levels of the inflammatory factors and AQP1 were measured by RT-qPCR and western blot analysis. The results (Fig. 5) revealed that the expression of the inflammatory factors, IL-1 $\beta$, IL- 6 and IL-8, was significantly downregulated in the presence of the p38 inhibitor (SB 203580), as well as in the presence of the ERK inhibitor (PD 98059). However, the JNK inhibitor (JNK inhibitor II) decreased only the expression level of IL-8, and no significant changes were observed in the expression of inflammatory factors in the presence of the PI3K inhibitor (LY 294002). These results indicate that PBEF regulates the expression of inflammatory factors and that the MAPK pathways, particularly p38 MAPK, are involved. The expression of AQP1 was upregulated significantly by the p38, ERK and JNK inhibitors, indicating that the regulatory effects of PBEF on AQP1 are dependent on the MAPK pathways. Taken together, our results suggest that PBEF regulates the expression of inflammatory factors and AQPs through the MAPK pathways.

\section{Discussion}

ARDS is still one of the main causes of death of patients in the intensive care unit (ICU), with a mortality rate of $40-60 \%$ (1); it is proving difficult to reduce the incidence and mortality rate of ARDS. The prevention of ALI is the main measure which must be taken in order to reduce the incidence of ARDS. There have 
A

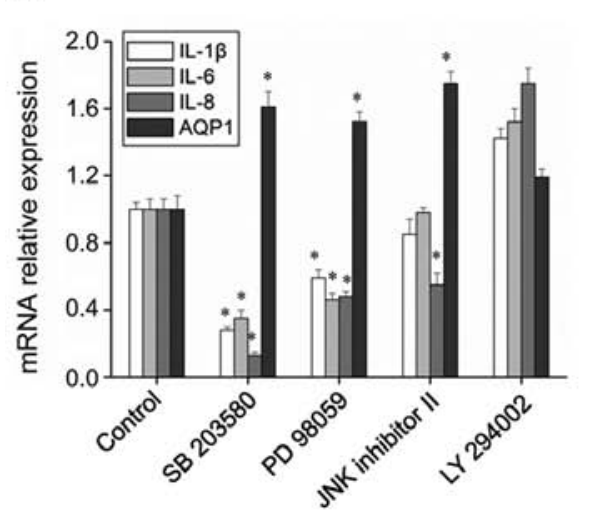

B

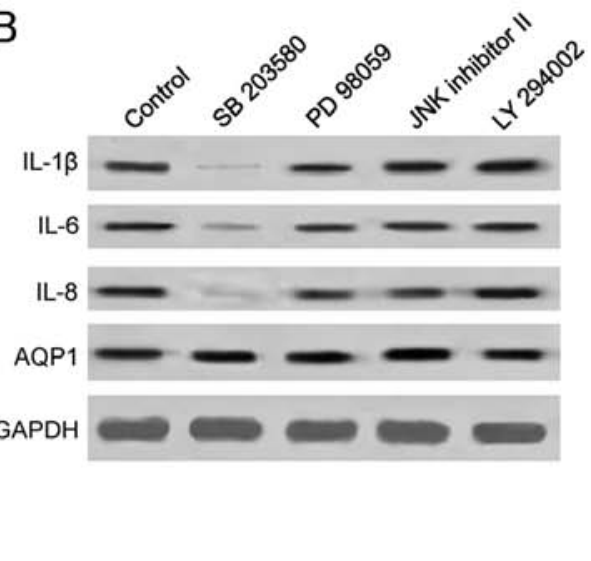

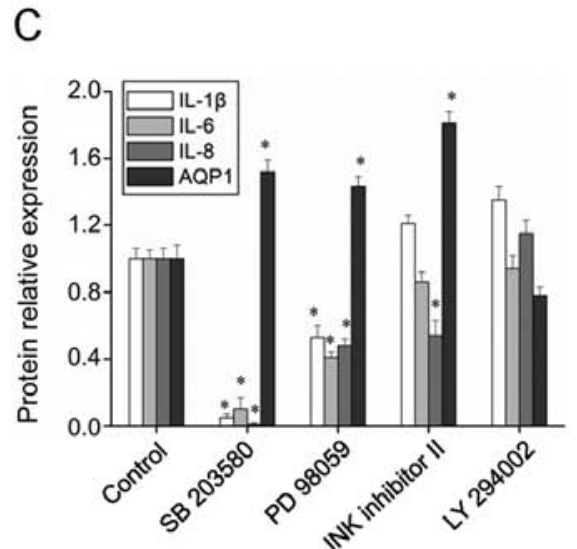

Figure 5. Effects of pre-B cell colony-enhancing factor (PBEF) overexpression on inflammatory factor and aquaporin (AQP)1 expression. Human pulmonary microvascular endothelial cells (HPMECs) were transfected with the PBEF-overexpressing plasmid (pEGFP-N1-PBEF) and then exposed to different signaling inhibitors, which include SB 203580 for p38, c-Jun N-terminal kinase (JNK) inhibitor II, PD 98059 for extracellular signal-regulated kinase (ERK) and LY 294002 for phosphoinositide 3-kinase (PI3K). Cells were exposed to the inhibitors at a final concentration of $20 \mu \mathrm{M}$ for $1 \mathrm{~h}$ prior to transfection and the induction of PBEF expression. DMSO was used as a negative control. (A) mRNA epxression; (B and C) protein expression.Each bar represents the means \pm SD from 3 samples ( $\mathrm{P}<0.05$ vs. control)

been many important developments in the study of the pathogenesis, treatment strategies and other aspects of ALI $(17,18)$. Studies have emphasized the important role which PBEF plays in the development of ALI. For instance, Kamp et al (19) proved that PBEF is a candidate gene which is related to ALI through genome-wide association studies (GWAS). The present study focused on investigating the important role of PBEF in ALI and its association with inflammatory signaling pathways and pulmonary water transport. Our results revealed that TNF- $\alpha$ promoted the expression of PBEF in HPMECs, which was consistent with the results of Hector et al (14). Moreover, Wang et al (20) previously reported that TNF- $\alpha$ enhanced the hyperbaric oxygen-induced visfatin expression through the JNK pathway in human coronary arterial endothelial cells.

It has been demonstrated that endothelial cell injury is an important marker of ALI, and that the degree of injury is closely related to the prognosis of ALI patients (21). Therefore, the study of the link between HPMEC dysfunction and ALI has attracted increasing attention. In the present study, the influence of PBEF overexpression on HPMEC apoptosis was detected by flow cytometry, and the results revealed that PBEF overexpression promoted HPMEC apoptosis, which was consistent with the findings of the study by Martin et al (22) and de Souza et al (23). In addition, Martin et al (22) found that ALI was related to an increase in cell apoptosis in lung tissue, a delay in inflammatory cell apoptosis, and the exacerbation of lung injury. However, during the healing process, the apoptosis of proliferous endothelial cells and fibroblasts occurred, and the lung tissue was reconstructed. de Souza et al (23) found that the increase in endothelial/epithelial cell apoptosis and the decrease in inflammatory cell apoptosis were closely related to the occurrence of ALI. Gao et al (24) also demonstrated that PBEF silencing using siRNA inhibited the expression of inflammatory cytokines and decreased the apoptosis mediated by FasL in HPMECs.

It has also been demonstrated that PBEF plays an important role in inflammatory signaling pathways and endothelial cell permeability; however, the exact mechanisms remain unclear (25). In the present study, we demonstrated that PBEF promoted the expression levels of the inflammatory factors, IL-1 $\beta$, IL-6 and IL-8, which resulted in the enhancement of the inflammatory response. Liu et al (6) and Li et al (26) proved that PBEF promoted the expression of IL-8 in human pulmonary alveolar epithelial cells. Ognjanovic et al (27) indicated that recombinant PBEF increased the expression of IL-6 and IL-8 in amniotic epithelial cells. PBEF has also been found to induce the mRNA and protein expression of IL-1, IL-6, IL-10 and TNF- $\alpha$ in PBMCs and that of IL- $1 \beta$, IL- 6 and TNF- $\alpha$ in CD14 monocytes (2). Therefore, PBEF and some corresponding inflammatory factors may have mutually promoting effects and may exhibit a 'waterfall-like' chain reaction in HPMECs. Our results confirmed that PBEF inhibited the expression of AQP1 in HPMECs. AQP1 is a crucial molecule in maintaining the balance of water movement between blood vessels and interstitial fluid (9). In a previous study using an animal model of lipopolysaccharide-induced ALI, the expression of AQP1 was decreased, and pneumonedema with ALI aggravation were observed (12). In a murine model of pneumonedema induced by viral infection, it was found that the expression of AQP1 and AQP5 in the mouse lungs was decreased, which then recovered when inflammation became slighter (11).

The mechanisms of the PBEF-regulated inflammatory response remain elusive. In the present study, we found that PBEF regulated the expression of inflammatory factors mainly through the MAPK pathways, which was consistent with the conclusion drawn by Moschen et al (2). However, they also found that PBEF regulated the expression of IL-10 and TNF- $\alpha$ through the PI3K pathway. Liu et al (7) demonstrated that the visfatin-induced monocyte chemoattractant protein (MCP)-1 and IL- 6 production involved the $\mathrm{p} 38$ MAPK, PI3K and ERK1/2 pathways in human umbilical vein endothelial cells. They also found that PBEF induced the expression of IL-8, IL-16 and CCR3 through the p38 and JNK pathways in pulmonary epithelial cells. Taken together, these results suggest that p38 MAPK is the main pathway through which $\mathrm{PBEF}$ regulates inflammatory factors.

The molecular mechanisms through which PBEF affects the expression of AQP1 have yet to be clarified. In this study, we found that the expression of AQP1 increased after blocking 
p38, ERK and JNK, which indicated that PBEF regulates the expression of AQP1 mainly through the MAPK pathways. Therefore, it may be inferred that, in the pathological process, the increase in PBEF expression inhibits AQP1 expression and causes pneumonedema, mainly through the MAPK pathways. It was also confirmed in this study that the MAPK pathways have a close association with AQP1. Similarly, Umenishi et al (28) demonstrated that the activation of the ERK, p38 and JNK pathways, as well as the hypertonicity response element in the AQP1 promoter, was involved in hypertonicity-induced AQP1 expression in mouse inner-medullary collecting duct (mIMCD)-3 cells. MEK/ERK mediated the UVB-induced AQP1 downregulation and water-permeability impairment in human retinal pigment epithelial cells, which was also confirmed by Jiang et al (29). Furthermore, Shankardas et al (30) found that AQP1 played an important role in human corneal endothelial cell (HCEC) proliferation and migration through the ERK signaling pathway.

In conclusion, our study demonstrated that PBEF regulated the pathological process of ALI by promoting HPMEC apoptosis and regulating the expression of inflammatory factors and AQP1 through the MAPK and PI3K pathways. These results provide valuable experimental evidence which may be used in future studies on the biological function, molecular mechanisms and clinical application potential of PBEF in ALl pathogenesis.

\section{Acknowledgements}

This study was supported by the Hunan Provincial Natural Science Foundation of China (2015JJ4056), Hunan Provincial Science and Technology Plan Project of China (2013FJ4106) and the High Technology Industry Department, Development and Reform Commission of Hunan Province, China (2012-1493).

\section{References}

1. Ware LB and Matthay MA: The acute respiratory distress syndrome. N Engl J Med 342: 1334-1349, 2000.

2. Moschen AR, Kaser A, Enrich B, Mosheimer B, Theurl M, Niederegger $\mathrm{H}$ and Tilg $\mathrm{H}$ : Visfatin, an adipocytokine with proinflammatory and immunomodulating properties. J Immunol 178 : 1748-1758, 2007.

3. Ye SQ, Simon BA, Maloney JP, Zambelli-Weiner A, Gao L, Grant A, Easley RB, McVerry BJ, Tuder RM, Standiford T, et al: Pre-B-cell colony-enhancing factor as a potential novel biomarker in acute lung injury. Am J Respir Crit Care Med 171: 361-370, 2005.

4. Bajwa EK, Yu CL, Gong MN, Thompson BT and Christiani DC: Pre-B-cell colony-enhancing factor gene polymorphisms and risk of acute respiratory distress syndrome. Crit Care Med 35: 1290-1295, 2007.

5. Ye SQ, Zhang LQ, Adyshev D, Usatyuk PV, Garcia AN, Lavoie TL, Verin AD, Natarajan V and Garcia JG: Pre-B-cell-colonyenhancing factor is critically involved in thrombin-induced lung endothelial cell barrier dysregulation. Microvasc Res 70: 142-151, 2005.

6. Liu P, Li H, Cepeda J, Zhang LQ, Cui X, Garcia JG and Ye SQ: Critical role of PBEF expression in pulmonary cell inflammation and permeability. Cell Biol Int 33: 19-30, 2009.

7. Liu SW, Qiao SB, Yuan JS and Liu DQ: Visfatin stimulates production of monocyte chemotactic protein-1 and interleukin-6 in human vein umbilical endothelial cells. Horm Metab Res 41: 281-286, 2009

8. Liu P, Li H, Cepeda J, Xia Y, Kempf JA, Ye H, Zhang LQ and Ye SQ: Regulation of inflammatory cytokine expression in pulmonary epithelial cells by pre-B-cell colony-enhancing factor via a nonenzymatic and AP-1-dependent mechanism. J Biol Chem 284: 27344-27351, 2009.
9. Verkman AS: Role of aquaporins in lung liquid physiology. Respir Physiol Neurobiol 159: 324-330, 2007.

10. Li J, Xu M, Fan Q, Xie X, Zhang Y, Mu D, Zhao P, Zhang B, Cao F, Wang Y, et al: Tanshinone IIA ameliorates seawater exposure-induced lung injury by inhibiting aquaporins (AQP) 1 and AQP5 expression in lung. Respir Physiol Neurobiol 176: 39-49, 2011.

11. Towne JE, Harrod KS, Krane CM and Menon AG: Decreased expression of aquaporin (AQP)1 and AQP5 in mouse lung after acute viral infection. Am J Respir Cell Mol Biol 22: 34-44, 2000.

12. Su X, Song Y, Jiang J and Bai C: The role of aquaporin-1 (AQP1) expression in a murine model of lipopolysaccharide-induced acute lung injury. Respir Physiol Neurobiol 142: 1-11, 2004.

13. Lai KN, Leung JC, Metz CN, Lai FM, Bucala R and Lan HY: Role for macrophage migration inhibitory factor in acute respiratory distress syndrome. J Pathol 199: 496-508, 2003.

14. Hector J, Schwarzloh B, Goehring J, Strate TG, Hess UF, Deuretzbacher G, Hansen-Algenstaedt N, Beil FU and Algenstaedt P: TNF-alpha alters visfatin and adiponectin levels in human fat. Horm Metab Res 39: 250-255, 2007.

15. Fujita-Yoshigaki J, Matsuki-Fukushima $M$ and Sugiya H: Inhibition of Src and p38 MAP kinases suppresses the change of claudin expression induced on dedifferentiation of primary cultured parotid acinar cells. Am J Physiol Cell Physiol 294: C774-C785, 2008.

16. Schindler JF, Monahan JB and Smith WG: p38 pathway kinases as anti-inflammatory drug targets. J Dent Res 86: 800-811, 2007.

17. Raghavendran K, Pryhuber GS, Chess PR, Davidson BA, Knight PR and Notter RH: Pharmacotherapy of acute lung injury and acute respiratory distress syndrome. Curr Med Chem 15: 1911-1924, 2008.

18. Licker M, Fauconnet P, Villiger Y and Tschopp JM: Acute lung injury and outcomes after thoracic surgery. Curr Opin Anaesthesiol 22: 61-67, 2009.

19. Kamp R, Sun X and Garcia JG: Making genomics functional: deciphering the genetics of acute lung injury. Proc Am Thorac Soc 5: 348-353, 2008.

20. Wang BW, Lin CM, Wu GJ and Shyu KG: Tumor necrosis factor- $\alpha$ enhances hyperbaric oxygen-induced visfatin expression via JNK pathway in human coronary arterial endothelial cells. J Biomed Sci 18: 27, 2011.

21. Maniatis NA, Kotanidou A, Catravas JD and Orfanos SE: Endothelial pathomechanisms in acute lung injury. Vascul Pharmacol 49: 119-133, 2008.

22. Martin TR, Nakamura $M$ and Matute-Bello G: The role of apoptosis in acute lung injury. Crit Care Med 31 (4 Suppl): S184-S188, 2003

23. de Souza PM and Lindsay MA: Apoptosis as a therapeutic target for the treatment of lung disease. Curr Opin Pharmacol 5: 232-237, 2005.

24. Gao W, Mao Q, Feng AW, Sun HM, Sun WK, Lu X, Su X and Shi Y: Inhibition of pre-B cell colony-enhancing factor attenuates inflammation and apoptosis induced by pandemic H1N1 2009 in lung endothelium. Respir Physiol Neurobiol 178: 235-241, 2011.

25. Pilz S, Mangge H, Obermayer-Pietsch B and März W: Visfatin/pre-B-cell colony-enhancing factor: a protein with various suggested functions. J Endocrinol Invest 30: 138-144, 2007.

26. Li H, Liu P, Cepeda J, Fang D, Easley RB, Simon BA, Zhang LQ and Ye SQ: Augmentation of pulmonary epithelial cell IL-8 expression and permeability by pre-B-cell colony enhancing factor. J Inflamm (Lond) 5: 15, 2008.

27. Ognjanovic S and Bryant-Greenwood GD: Pre-B-cell colony-enhancing factor, a novel cytokine of human fetal membranes. Am J Obstet Gynecol 187: 1051-1058, 2002.

28. Umenishi F and Schrier RW: Hypertonicity-induced aquaporin-1 (AQP1) expression is mediated by the activation of MAPK pathways and hypertonicity-responsive element in the AQP1 gene. J Biol Chem 278: 15765-15770, 2003.

29. Jiang Q, Cao C, Lu S, Kivlin R, Wallin B, Chu W, Bi Z, Wang X and Wan Y: MEK/ERK pathway mediates UVB-induced AQP1 downregulation and water permeability impairment in human retinal pigment epithelial cells. Int J Mol Med 23: 771-777, 2009.

30. Shankardas J, Patil RV and Vishwanatha JK: Effect of down-regulation of aquaporins in human corneal endothelial and epithelial cell lines. Mol Vis 16: 1538-1548, 2010. 